\title{
Nutritional upregulation of $p 85 \alpha$ expression is an early molecular manifestation of insulin resistance
}

Received: 15 September 2005 / Accepted: 9 November 2005 / Published online: 21 February 2006

(C) Springer-Verlag 2006

\begin{abstract}
Aims/hypothesis: We sought to define early molecular alterations associated with nutritionally induced insulin resistance in humans. Methods: Insulin sensitivity was assessed using a hyperinsulinaemic-euglycaemic clamp in eight healthy women while on an isocaloric diet and after 3 days of overfeeding (50\% above eucaloric diet). Expression of phosphatidylinositol (PI) 3-kinase subunits $\mathrm{p} 85 \alpha$ and $\mathrm{p} 110$ was assessed and measurements were made of IRS-1-associated PI 3-kinase activity, tyrosine and serine phosphorylation of IRS-1, and serine and threonine phosphorylation of p70S6 kinase. Measurements were made in skeletal muscle biopsies obtained before and after overfeeding. Results: Three days of overfeeding resulted in a reduction of insulin sensitivity accompanied by: (1) increased expression of skeletal muscle p $85 \alpha$; (2) an alteration in the ratio of $p 85 \alpha$ to p110; (3) a decrease in the amount of IRS-1-associated p110; and (4) a decrease in PI 3-kinase activity. Increases in expression of $p 85 \alpha$ and in the $p 85 \alpha: p 110$ ratio demonstrated a highly significant inverse correlation with insulin sensitivity, and changes in PI 3-kinase activity correlated with changes in insulin sensitivity. Tyrosine and serine phosphorylation of IRS-1 and serine and threonine phosphorylation of p70S6 kinase were unaffected by 3 days of overfeeding. Conclusions/interpretation: We identified a novel mechanism of nutritionally induced insulin resistance in healthy women of normal weight. We conclude that increased expression of $p 85 \alpha$ may be one
\end{abstract}

M.-A. Cornier · D. H. Bessesen · I. Gurevich · J. W. Leitner

B. Draznin

Research Service, Department of Veterans Affairs,

and the Department of Medicine,

University of Colorado Health Sciences Center,

Denver, CO, USA

B. Draznin $(\bowtie)$

VA Hospital (151)

1055 Clermont Street,

Denver, CO 80220, USA

e-mail: boris.draznin@med.va.gov

Tel.: +1-303-3934619

Fax: $+1-303-3775686$ of the earliest molecular alterations in the mechanism of the insulin resistance associated with overfeeding.

Keywords Insulin resistance - Insulin signalling • Overfeeding · Phosphatidylinositol 3-kinase

Abbreviations GCRC: General Clinical Research Center - mTOR: molecular target of rapamycin PI 3-kinase: phosphatidylinositol 3-kinase

\section{Introduction}

Insulin resistance is a prevalent and well-defined clinical condition associated with obesity, type 2 diabetes mellitus, hypertension and the metabolic syndrome $[1,2]$, but its primary cause remains unknown. At the cellular level, metabolic insulin resistance displays a reduced strength of signalling via the IRS-phosphatidylinositol (PI) 3-kinase pathway, accompanied by a reduction in glucose uptake and utilisation [3].

At least two complementary mechanisms have recently emerged as a potential explanation for the reduced strength of the IRS-PI 3-kinase signalling pathway. First, it became apparent that serine phosphorylation of IRS proteins either by PI 3-kinase itself, or by protein kinase C, by a mechanism dependent on molecular target of rapamycin (mTOR), or by other kinases, can reduce the ability of IRS proteins to attract PI 3-kinase, thus minimising its activation [4-8]. Under certain conditions, excessive serine phosphorylation of IRS proteins could become detrimental to normal insulin signalling downstream.

A second possibility is a disruption of the balance between the amounts of the PI 3-kinase subunits [9]. This enzyme consists of a regulatory subunit, p85, and a catalytic subunit, p110 (reviewed in [10]). Normally, the regulatory subunit exists in stoichiometric excess to the catalytic one, resulting in a pool of free p 85 monomers not associated with the p110 catalytic subunit. The p85 monomers bind to phosphorylated IRS proteins, blocking access to p $85-\mathrm{p} 110$ heterodimers. Thus, there is a balance 
between the free $\mathrm{p} 85$ monomer and the $\mathrm{p} 85-\mathrm{p} 110$ heterodimer, the latter being responsible for the PI 3-kinase activity. Increases or decreases in expression of p85 shift this balance in favour of either free p85 or p85-p110 complexes [11-14]. Because the monomer and the heterodimer compete for the same binding sites on the IRS proteins, an imbalance could cause either increased or decreased PI 3-kinase activity. This possibility has recently been supported by studies of the insulin-resistant states induced by human placental growth hormone [15], obesity and type 2 diabetes [16]. If the production of $\mathrm{p} 85$ can be enhanced nutritionally, the resulting changes in the ratio of p85 to p110 may be the earliest manifestation of the ensuing insulin resistance. This could also explain how overfeeding and weight gain trigger insulin resistance.

To determine whether serine phosphorylation of IRS-1 and/or an alteration in expression of the subunits of PI 3kinase play a role in the mechanism of nutritionally induced insulin resistance, we assessed whole-body insulin sensitivity, the expression of the subunits of PI 3-kinase, the activity of PI 3-kinase, serine and tyrosine phosphorylation of IRS-1 and serine phosphorylation of p70S6 kinase in skeletal muscle of eight healthy women of normal weight before and after 3 days of overfeeding at $50 \%$ above the basal energy requirement.

\section{Subjects, materials and methods}

\section{Subjects}

Eight non-diabetic, healthy women of normal weight (BMI $20.6 \pm 1.8 \mathrm{~kg} / \mathrm{m}^{2}$ ) were enrolled in the study (Table 1 ). The study was approved by the Colorado Multiple Institutional Review Board, and all subjects gave informed consent.

\section{Materials}

Bovine serum albumin (BSA) and protease inhibitors aprotinin and leupeptin were purchased from Boehringer Mannheim (Indianapolis, IN, USA). Antibodies to IRS-1, total (pan) p85 and specific to the $\alpha$ component of $\mathrm{p} 85$

Table 1 Characteristics of the eight female subjects

\begin{tabular}{ll}
\hline Characteristic & Value \\
\hline Age (years) & $30.6 \pm 8.0$ \\
Body weight $(\mathrm{kg})$ & $54.9 \pm 6.1$ \\
BMI $\left(\mathrm{kg} / \mathrm{m}^{2}\right)$ & $20.6 \pm 1.8$ \\
Fat mass $(\mathrm{kg})$ & $14.1 \pm 3.6$ \\
Lean body mass $(\mathrm{kg})$ & $40.9 \pm 4.1$ \\
Glucose $(\mathrm{mmol} / \mathrm{l})$ & $4.31 \pm 0.26$ \\
Insulin $(\mathrm{pmol} / \mathrm{l})$ & $29.9 \pm 11.1$ \\
NEFA $(\mu \mathrm{Eq} / \mathrm{l})$ & $862 \pm 84$ \\
Glycerol $(\mu \mathrm{mol} / \mathrm{l})$ & $63.5 \pm 6.5$ \\
Leptin $(\mathrm{nmol} / \mathrm{l})$ & $0.31 \pm 0.09$ \\
\hline
\end{tabular}

Data are mean \pm SD (p85 $\alpha$ or PIK3R1) were purchased from Upstate Biotechnology (Lake Placid, NY, USA). Antibodies to p110 were purchased from Santa Cruz Biotechnology (Santa Cruz, CA, USA). Anti-phosphoserine IRS-1 antibody was from Upstate Biotechnology, pY-20 antibody was from BD Transduction Laboratories (San Diego, CA, USA) and antiphospho p70S6 kinase antibodies (Thr 3389 and Thr421/ Ser424) were from Cell Signaling Technology (Beverly, MA, USA). Secondary horseradish-peroxidase-conjugated antibody, protein A Sepharose and chemiluminescence reagents were obtained from Amersham Pharmacia Biotech (Piscataway, NJ, USA). Analytical grade resins, polyvinylidene difluoride membranes, PAGE gel equipment and protein assay kits were from Bio-Rad Laboratories (Hercules, CA, USA). $\left[\gamma^{-}{ }^{32} \mathrm{P}\right]$ ATP was obtained from NEN Life Science (Boston, MA, USA).

\section{Study design}

Subjects first underwent baseline assessments, including measurements of resting metabolic rate by hood indirect calorimetry (2,900 Metabolic Cart; Sensormedics, Yorba Linda, CA, USA) and body composition measurement by dual-energy X-ray absorptiometry (DPX whole-body scanner; Lunar Radiation, Madison, WI, USA). Subjects were then placed on a eucaloric diet for 3 days to ensure weight maintenance and energy balance (days 1-3). Estimates of daily energy needs were made using selfreported intake with a 3-day food diary, the HarrisBenedict equation, and baseline resting metabolic rate plus an activity factor. This was followed by the first study day (day 4). Subjects were then maintained on a eucaloric diet for 3 more days (days 5-7). On day 8, energy intake was increased by $50 \%$ (overfeeding diet) and this was continued for 3 days (days $8-10$ ). This was followed by the second study day (day 11). The macronutrient compositions of the diets were kept stable at $50 \%$ carbohydrate, $30 \%$ fat and $20 \%$ protein. The polyunsaturated:saturated fat ratio and fibre and cholesterol contents of the diets were identical. All food was prepared and provided by the General Clinical Research Center (GCRC) kitchen. Subjects presented to the GCRC every morning. They were weighed, ate breakfast, and picked up the remainder of their daily meals in coolers. Subjects were asked to maintain their usual pattern of physical activity and were questioned daily regarding activity and compliance. Subjects were asked not to consume any alcoholic or additional calorie-containing beverages during the study period.

Study days

Subjects were admitted to the GCRC the evening before the metabolic studies were performed (on the two study days, i.e. days 4 and 11). The morning after admission, following an overnight fast, a vastus lateralis skeletal 
muscle biopsy was performed. The biopsy samples were frozen immediately and stored at $-80^{\circ} \mathrm{C}$ until used.

Next, a euglycaemic-hyperinsulinaemic clamp study was performed. A standard antecubital venous catheter was placed in one arm for infusions, and a second catheter was placed retrograde in a dorsal hand vein of the contralateral arm for sampling, using the heated hand technique to obtain arterialised venous blood [17]. Blood was then sampled for eucaloric baseline measurements. Next, a primed, continuous infusion of insulin at $40 \mathrm{mU} \mathrm{m}^{-2} \mathrm{~min}^{-1}$ was initiated, and continued for $100 \mathrm{~min}$. Blood samples were taken at 80,90 and $100 \mathrm{~min}$ for steady-state measurements of metabolites. Plasma glucose was measured immediately upon sampling during the euglycaemichyperinsulinaemic clamp studies using a YSI glucose analyser [18] every $5 \mathrm{~min}$. A $20 \%$ dextrose solution was infused and adjusted to maintain euglycaemia at a blood glucose concentration of approximately $5 \mathrm{mmol} / \mathrm{l}$.

\section{Determination of IRS-1-associated PI 3-kinase activity}

Lysates prepared from the tissue biopsy were immunoprecipitated with IRS-1 antibody. PI 3-kinase activity was determined in 1-3 $\mu \mathrm{l}$ of the immunoprecipitate by thin layer chromatography, as described previously [15].

Measurements of total (pan-p85) and p85 $\alpha$

in the lysates

The lysates were immunoblotted with the pan-p85 antibody to determine the amounts of total $\mathrm{p} 85$ in the samples. Pan-p85 antibody interacts with all isoforms of p85, thus providing an accurate measure of the total amounts of all isoforms in the lysate. In order to measure quantitatively the amounts of $\mathrm{p} 85 \alpha$ (designated subsequently PIK3R 1 ), the lysates were immunoprecipitated with PIK3R1-specific antibody and immunoblotted with the same antibody. The remaining supernatants were immunoblotted to assure complete immunoprecipitation of this subunit. The differences in the amounts of total $\mathrm{p} 85$ and PIK3R1 represent the amounts of $\mathrm{p} 85 \beta, \mathrm{p} 55 \alpha$ and $\mathrm{p} 50$.

\section{Determination of $\mathrm{p} 110$}

Cell lysates were triple-immunoprecipitated with pan-p110 antibody and immunoblotted with the same pan-p110 antibody. The $\mathrm{p} 110$ precipitates and supernatants were also blotted with p85 $\alpha$ antibody. PIK3R1 associated with p110 denotes the amount of PIK3R1-p110 heterodimer, while PIK3R1 in the supernatant reflects the amount of free PIK3R1 monomer. Amounts of p110 were also determined in the IRS-1 immunoprecipitates.

Determination of phosphorylation of IRS-1 and p70S6 kinase

IRS-I immunoprecipitates were probed with pY20 antibody and with anti-serine 307 antibodies to determine the extent of tyrosine and serine phosphorylation of IRS-1. The post-IRS-1 supernatants were blotted with anti-p70S6 kinase Thr 421/Ser424 and Thr 386 antibodies to determine the mTOR-mediated phosphorylation of p70S6 kinase.

\section{Statistical analysis}

The data are expressed as mean \pm SEM. Statistical analysis was performed with SigmaStat statistical software (Jandel Scientific, San Rafael, CA, USA). The effects of overfeeding were analysed using the paired $t$ test; $p$ values of $<0.05$ were considered statistically significant. The relationships between whole-body insulin sensitivity and insulin signalling proteins were examined using the Pearson product correlation.
Fig. 1 a Representative immunoblots and bar graph of total (Pan)-p85, p85 $\alpha$ (PIK3R1), and p110 before $(b f)$ and after $(a f)$ 3 days of overfeeding. b Effects of 3 days of overfeeding on p85 $\alpha$ :p110 ratio in skeletal muscle of eight lean women. Results are expressed as mean and SEM. ${ }^{*} p<0.05$
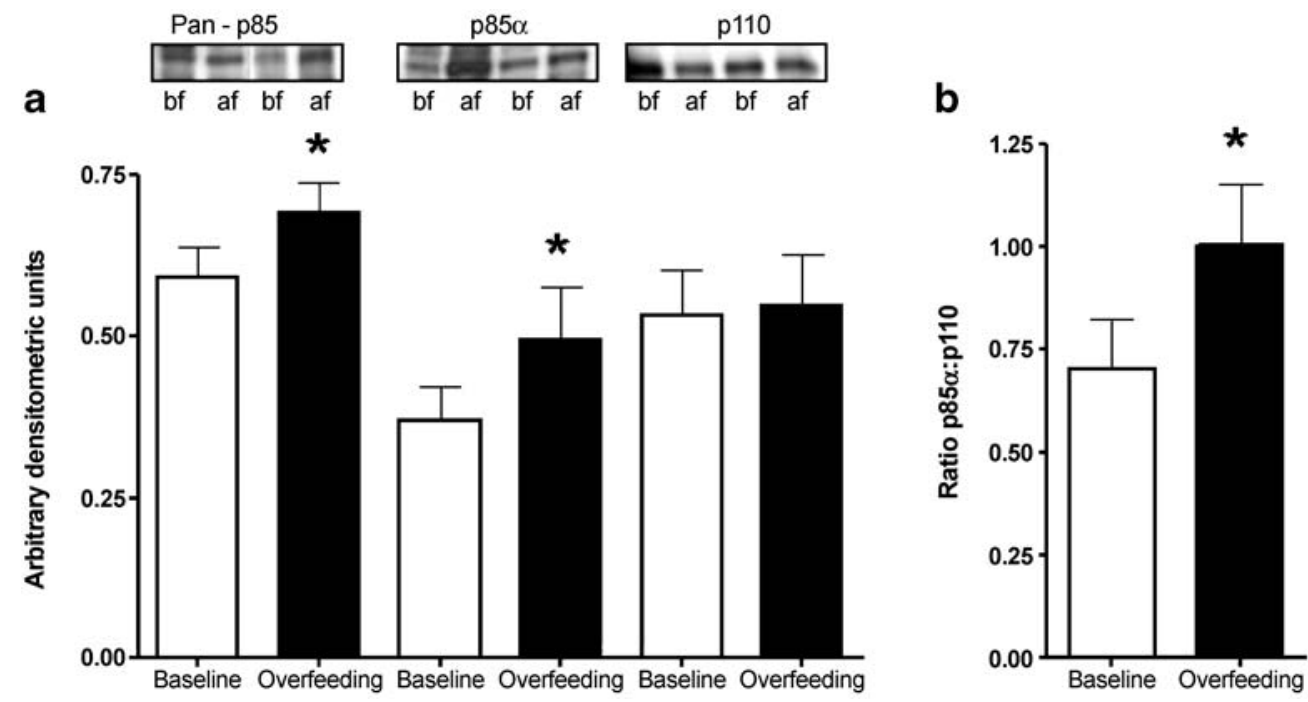


\section{Results}

Three days of overfeeding resulted in a reduction in wholebody insulin sensitivity, measured as the glucose infusion rate (from $12.46 \pm 1.43$ to $10.78 \pm 1.12 \mathrm{mg} \mathrm{kg}^{-1} \mathrm{~min}^{-1}$, $p=0.02$ ). Three days of overfeeding also resulted in significant increases in total p85 protein production by skeletal muscle (Fig. 1; $p=0.046$ ). This rise in the amount of total p85 was accounted for by a significant increase $(p=0.02)$ in PIK3R1 (Fig. 1). Overfeeding did not alter p110 expression, but significantly increased the ratio of PIK3R1 to p110 (Fig. $1 ; p=0.046$ ).

If the increasing amounts of free PIK3R1 compete with and displace the p85-p110 heterodimers from association with IRS-1, then both the amounts of p110 and the PI 3-kinase activity would be reduced in the IRS-1 immunoprecipitates. To investigate this possibility, we immunoprecipitated IRS-1 and determined the amounts of p110 and the PI 3-kinase activity in the immunoprecipitates. Both were significantly reduced by overfeeding (Fig. 2a,b).

Changes in the amount of insulin signalling intermediates correlated with changes in whole-body insulin sensitivity. Specifically, the PIK3R1:p110 ratio correlated inversely with insulin sensitivity $(r=-0.75, p=0.008$; Fig. 3a), while changes in the PI 3-kinase activity correlated with changes in insulin sensitivity $(r=0.52$, $p=0.05$; Fig. 3b), strongly suggesting that imbalance between the amount of PIK3R1 and p110 results in clinically apparent insulin resistance.

In contrast to the changes in PIK3R1and PI 3-kinase activity, 3 days of overfeeding did not alter serine or tyrosine phosphorylation of IRS-1, serine and threonine phosphorylation of p70S6 kinase (a direct substrate of mTOR) or the total amounts of IRS-1 and p70S6 kinase in the skeletal muscle of these individuals (Fig. 4), indicating that after 3 days of overfeeding serine phosphorylation of IRS-1 or its downstream signalling molecules is not likely to represent the mechanism of decreased insulin sensitivity.

\section{Discussion}

We found that caloric excess, even if of short duration, reduced insulin sensitivity in healthy women and increased the amount of PIK3R1 in their skeletal muscle, suggesting that an imbalance between p85 $\alpha$ (PIK3R1) and p110 may be the earliest molecular manifestation of insulin resistance elicited by positive energy balance.

Reduced glucose uptake and utilisation in the state of insulin resistance are accompanied by a diminished strength of signalling via the IRS-1-PI 3-kinase pathway [3]. Two potential mechanisms for the reduction in the strength of the PI 3-kinase-dependent signalling pathway have been identified. First, it appears that serine phosphorylation of IRS protein diminishes its ability to attract the subsequent layer of signalling intermediates, thereby diminishing insulin action [4-8]. Activation of protein kinase $\mathrm{C}$ or Akt can lead to serine phosphorylation of IRS proteins (Ser-307 in rodents and Ser-312 in humans). Because activation of $\mathrm{mTOR}$ and its target $\mathrm{p} 70 \mathrm{~S} 6$ kinase by nutrients can result in serine phosphorylation of IRS-1 and a reduction in the strength of the PI 3-kinase-dependent pathway, this mechanism has deservedly gained considerable acceptance [4-8, 19].

A cellular nutrient sensor, mTOR, has been identified as a critical element integrating cellular metabolism with growth factor signalling [20-23]. In response to insulin and amino acids, mTOR phosphorylates and modulates the activities of p70 S6 kinase and an inhibitor of translational initiation, eIF-4E-binding protein [24-26]. Whereas insulin activates mTOR and p70S6 kinase via the IRS-1-PI 3kinase-Akt pathway [27, 28], amino acids seem to exert their effect through a direct influence of Akt [29]. In any

a

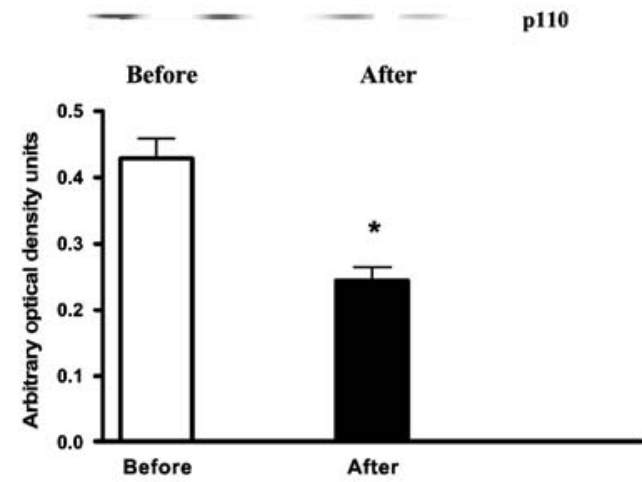

b

PI3-P
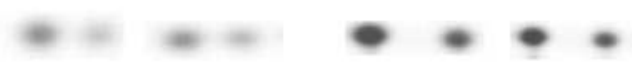

Origin

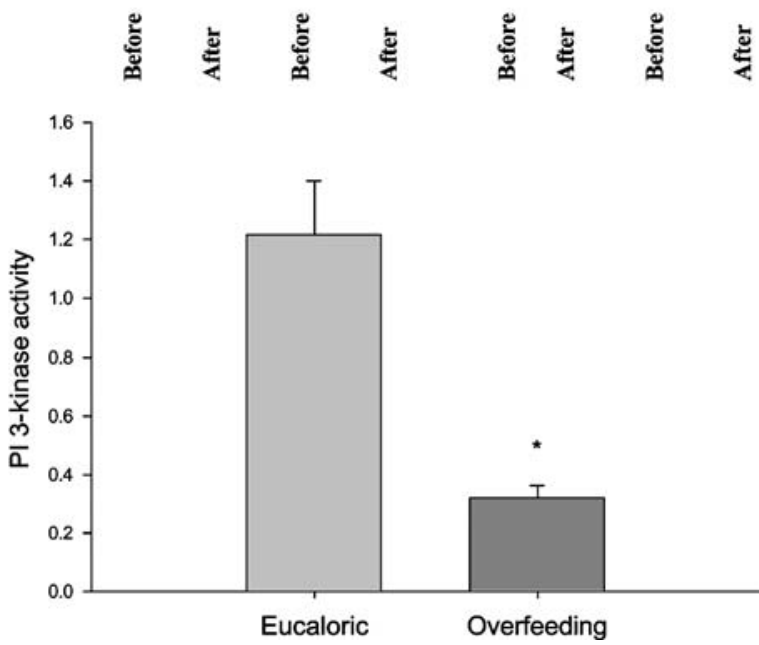

Fig. 2 Expression of p110 (a) and PI 3-kinase activity (b) in IRS-1 immunoprecipitates of skeletal muscle obtained from lean women before and after overfeeding. Upper panels depict representative blots. Results are expressed as mean and SEM. ${ }^{*} p<0.05$ 
event, activation of mTOR and p70S6 kinase causes increased serine phosphorylation of IRS-1 with a subsequent decline in IRS-1-associated PI 3-kinase activity.

Even though in vitro and ex vivo experiments have demonstrated an acute inhibitory effect of mTOR on insulin signalling in 3T3-L1 and human adipocytes [23], the present study in human muscle did not reveal an impairment in this pathway after 3 days of overfeeding. It has been noted previously that regulation of mTOR by amino acids in isolated adipocytes differs from that found in other tissues [30].

A second potential mechanism that can inhibit insulin action is an imbalance between the subunits of the PI 3kinase, i.e. the amounts of free $\mathrm{p} 85$ monomer and the $\mathrm{p} 85$ p110 heterodimer of the PI 3-kinase [9, 11-14]. PI 3-kinase belongs to the class 1a 3-kinases [10], which exist as heterodimers, and consists of a regulatory subunit (p85) that is tightly associated with a catalytic subunit, $\mathrm{p} 110$. The
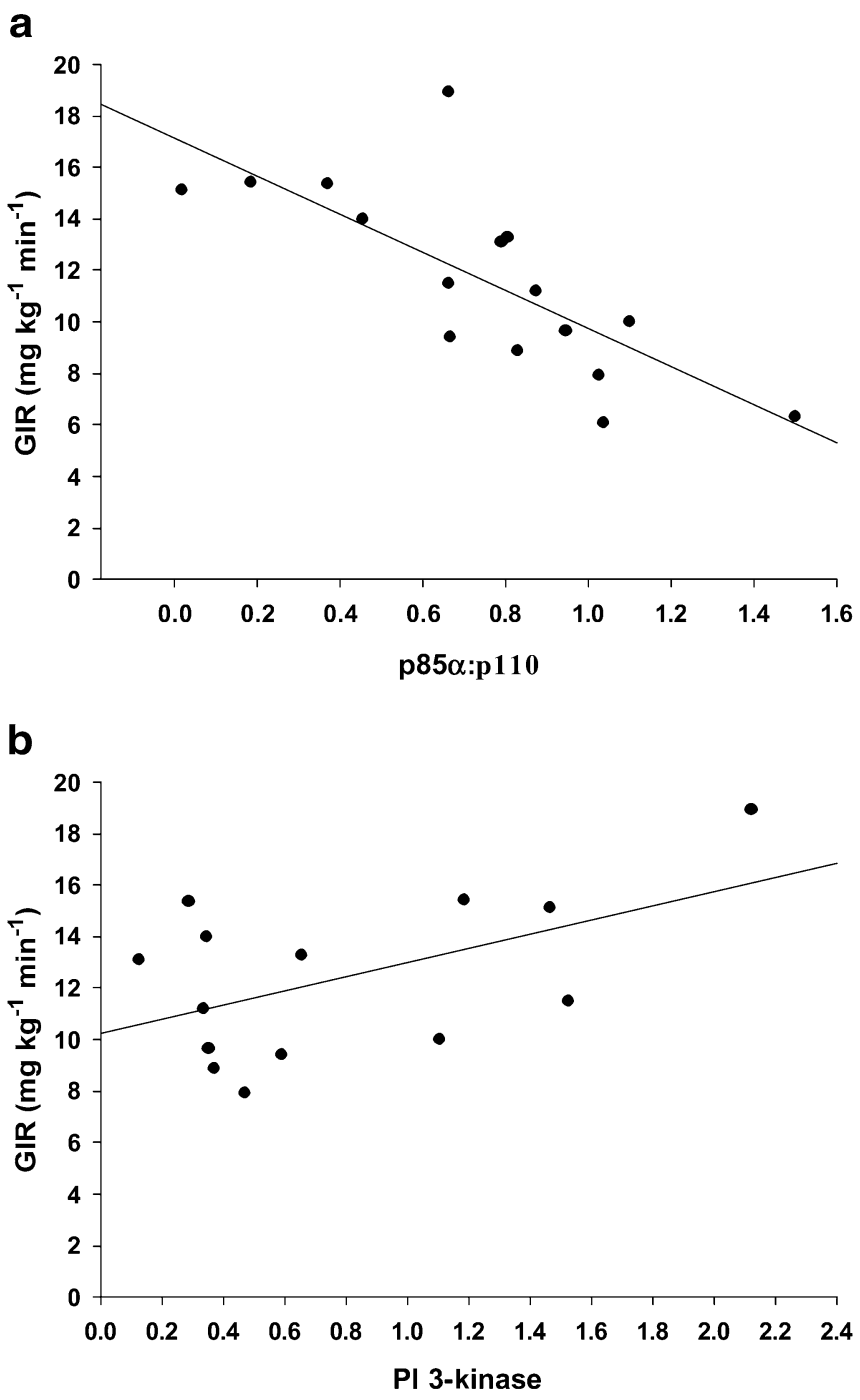

Fig. 3 Correlation between the p85 :p110 ratio and glucose infusion rate (GIR) (a) and changes in PI 3-kinase activity and changes in GIR (b) in eight lean women. $r=-0.75, p=0.008$ (a); $r=0.52, p=0.05$ (b) regulatory subunit, $\mathrm{p} 85$, is encoded by at least three genes that generate highly homologous products. Two isoforms are termed $\mathrm{p} 85 \alpha$ (PIK3R1) and $\mathrm{p} 85 \beta$ (products of the two genes). Three splice variants of PIK3R1 have been reported, including PIK3R1 itself, p55 $\alpha$ and p50 $\alpha$. The third gene product is $\mathrm{p} 55 \gamma$. The PIK3R1, however, appears to be the most abundant isoform [10].

One of the first indications that an imbalance between the amounts of p85 and p110 can alter PI 3-kinase activity came from the experiments with cultured skeletal muscle L6 cells treated with dexamethasone [31]. This treatment significantly reduced PI 3-kinase activity, despite almost a four-fold increase in the expression of PIK3R1 (no change in p $85 \beta$ ) and only a minimal increase in p110. The authors concluded that PIK3R1 competes with p85-p110 heterodimer, thus reducing PI 3-kinase activity.

Subsequently, animals with a targeted disruption of $p 85 \alpha\left(\mathrm{p} 85^{+/-}\right.$heterozygous mice) were found to have a higher ratio of $p 85-\mathrm{p} 110$ dimer to free $\mathrm{p} 85$ and to be more sensitive to insulin $[9,11-14,32-34]$. In order to determine this ratio, the authors immunodepleted p110 and blotted both the immunoprecipitates and the supernatant with $\mathrm{p} 85$ antibody. The amounts of p 85 in the p110 immunoprecipitates denote $\mathrm{p} 85$ bound to $\mathrm{p} 110$, while the amount of $\mathrm{p} 85$ in the supernatant represents free (excess) p85. The greater the ratio of bound to free $\mathrm{p} 85$, the greater insulin sensitivity mice display. The same group of authors then overexpressed PIK3R1 in cultured cells. This overexpression significantly inhibited PI 3-kinase activity [12]. Overexpression of $\mathrm{p} 50 \alpha$ or $\mathrm{p} 55 \alpha$ did not inhibit PI 3-kinase activity to the same extent. These experimental results are consistent with the competition hypothesis.

Recently, Barbour et al. [15] demonstrated that the insulin resistance of pregnancy is likely to be due to

a

IRS-1

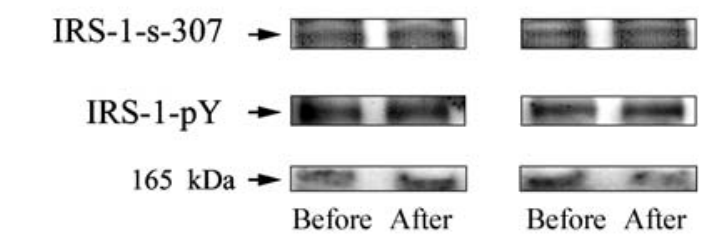

b

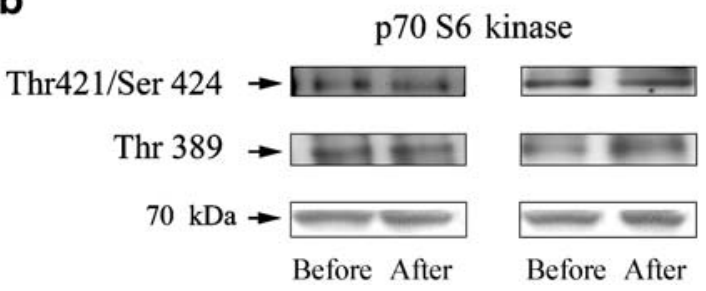

Fig. 4 Representative blots of a IRS-1-serine-307 phosphorylation, tyrosine phosphorylation $(p Y)$ and total IRS-1 (165 kDa) and b serine/threonine phosphorylation and total p70 S6 kinase. Results from skeletal muscle biopsy obtained from two lean women before and after overfeeding are depicted. None of the eight women studied demonstrated any differences either in the amount or in phosphorylation of IRS-1 or p70S6 kinase before and after overfeeding 
increased expression of the skeletal muscle $\mathrm{p} 85$ in response to increasing concentrations of human placental growth hormone. Furthermore, women remaining insulin-resistant post-partum have been found to display higher levels of p85 in muscle [35]. Thus, results reported in the literature support the hypothesis that $\mathrm{p} 85$ monomer completes with a p85-p110 dimer and that removal of the excess of p85 improves insulin sensitivity by allowing the remaining isoforms to bring p110 to its site of action.

Even though 3 days of overfeeding did not alter the serine phosphorylation of IRS-1 and p70S6 kinase, it is conceivable that excessive serine phosphorylation of these signalling molecules can cooperate with an imbalance between the PI 3-kinase subunits in the genesis of nutritionally induced insulin resistance, but the imbalance appears to be the initial step. It is also plausible that for individuals to develop clinically significant insulin resistance leading to glucose intolerance and diabetes, both abnormalities must be present: excessive serine phosphorylation of IRS-1 (stimulated either by insulin itself or by amino acids via the mTOR-p70S6 kinase feedback) and an increase in the expression of PIK3R1 enhanced by energy excess. These two defects are likely to complement one another in the genesis of insulin resistance. This possibility is supported by the recently reported findings that increased expression of PIK3R1 and impaired PI 3-kinase activity were associated with increased serine phosphorylation of IRS-1 and increased activity of proinflammatory kinases in skeletal muscle of obese subjects and subjects with type 2 diabetes [16]

In summary, our data describe a novel mechanism whereby a short period of overfeeding can impair insulin sensitivity in healthy lean women by enhancing the expression of PIK3R1 and altering the ratio of PIK3R1 to $\mathrm{p} 110$. The resulting excess of PIK3R1 monomer competes with PIK3R1-p110 dimer and reduces PI 3kinase activity. Thus, PIK3R1 may be a potential therapeutic target for the treatment and prevention of insulin resistance.

Acknowledgements This work was supported by the Veterans Affairs Research Service, the General Clinical Research Center, DK 48520, the National Center for Research Resources (M.-A. Cornier), RR16185, and the Foundation for Biomedical Education and Research.

\section{References}

1. Reaven GM (1998) Role of insulin resistance in human disease. Diabetes 37:1595-1607

2. Ginsberg H (2000) Insulin resistance and cardiovascular disease. J Clin Invest 106:453-458

3. Shulman GI (1999) Cellular mechanisms of insulin resistance in humans. Am J Cardiol 84:3J-10J

4. Qiao L, Goldberg JL, Russell JC, Sun XJ (1999) Identification of enhanced serine kinase activity in insulin resistance. J Biol Chem 274:10625-10632

5. White MF (2003) Insulin signaling in health and disease. Science 302:1710-1711

6. Birnbaum MJ (2001) Turning down insulin signaling. J Clin Invest 108:655-659
7. Um SH, Frigerio F, Watanabe M et al (2004) Absence of S6K1 protects against age- and diet-induced obesity while enhancing insulin sensitivity. Nature 431:200-205

8. Patti M-E, Kahn BB (2004) Nutrient sensor links obesity with diabetes risk. Nat Med 10:1049-1050

9. Ueki K, Fruman DA, Brachmann SM, Tseng YH, Cantley LC, Kahn CR (2002) Molecular balance between the regulatory and catalytic subunits of phosphoinositide 3-kinase regulates cell signaling and survival. Mol Cell Biol 22:965-977

10. Shepherd PR, Withers DJ, Siddle K (1998) Phosphoinositide 3kinase: the key switch mechanism in insulin signaling. Biochem J 333:471-490

11. Terauchi Y, Tsuji Y, Satoh S et al (1999) Increased insulin sensitivity and hypoglycaemia in mice lacking the p85a subunit of phosphoinositide 3-kinase. Nat Genet 21:230-235

12. Ueki K, Algenstaedt P, Mauvais-Jarvis F, Kahn CR (2000) Positive and negative regulation of phosphoinositide 3-kinasedependent signaling pathways by three different gene products of the p85 $\alpha$ regulatory subunit. Mol Cell Biol 20:8035-8046

13. Mauvais-Jarvis F, Ueki K, Fruman DA et al (2002) Reduced expression of the murine $\mathrm{p} 85 \alpha$ subunit of phosphoinositide 3kinase improves insulin signaling and ameliorates diabetes. J Clin Invest 109:141-149

14. Ueki K, Fruman DA, Uballe CM et al (2003) Positive and negative roles of $\mathrm{p} 85 \alpha$ and $\mathrm{p} 85 \beta$ regulatory subunits of phosphoinositide 3-kinase in insulin signaling. $\mathrm{J}$ Biol Chem 278:48453-48466

15. Barbour LA, Shao J, Qiao L et al (2004) Human placental growth hormone increases expression of $\mathrm{p} 85$ regulatory unit of phosphatidylinositol 3-kinase and triggers severe insulin resistance in skeletal muscle. Endocrinology 145:1144-1150

16. Bandyopadhyay GK, Yu JG, Ofrecio J, Olefsky JM (2005) Increased p85/55/50 expression and decreased phosphatidylinositol 3-kinase activity in insulin-resistant human skeletal muscle. Diabetes 54:2351-2359

17. Jensen MD, Heiling VJ (1991) Heated hand vein blood is satisfactory for measurements during free fatty acid kinetic studies. Metabolism 40:406-409

18. Kadish AH, Hall DA (1965) A new method for the continuous monitoring of blood glucose by measurement of dissolved oxygen. Clin Chem 11:869-875

19. Tremblay F, Krebs M, Dombrowski L et al (2005) Overactivation of S6 kinase 1 as a cause of human insulin resistance during increased amino acid availability. Diabetes $54: 2674-2684$

20. Raught B, Gingras AC, Sonenberg N (2001) The target of rapamycin (TOR) proteins. Proc Natl Acad Sci USA 98: 7037-7044

21. Rohde J, Heitman J, Cardenas ME (2001) The TOR kinases link nutrient sensing to cell growth. J Biol Chem 276:9583-9586

22. Khamzina L, Veilleux A, Bergeron S, Marette A (2005) Increased activation of the mammalian target of rapamycin pathway in liver and skeletal muscle of obese rats: possible involvement in obesity-linked insulin resistance. Endocrinology 146:1473-1481

23. Trembley F, Gagnon A, Veilleux A, Sorisky A, Marette A (2005) Activation of the mammalian target of rapamycin pathway acutely inhibits insulin signaling to Akt and glucose transport in 3T3-L1 and human adipocytes. Endocrinology 146:1328-1337

24. Burnett PE, Barrow RK, Cohen NA, Snyder SH, Sabatini DM (1998) RAFT1 phosphorylation of the translational regulators p70S6 kinase and 4E-BP1. Proc Natl Acad Sci USA 95:1432-1437

25. Hara K, Yonezawa K, Kozlowski MT et al (1997) Regulation of eIF-4E BP1 phosphorylation by mTOR. J Biol Chem 272:26457-26463

26. Isotani S, Hara K, Tokunaga C, Inoue H, Avruch J, Yonezawa K (1999) Immunopurified mammalian target of rapamycin phosphorylates and activates p70S6 kinase $\alpha$ in vitro. J Biol Chem 274:34493-34498 
27. Nave BT, Ouwens M, Withers DJ, Alessi DR, Shepherd PR (1999) Mammalian target of rapamycin is a direct target for protein kinase B: identification of a convergence point for opposing effects of insulin and amino-acid deficiency on protein translation. Biochem J 344:427-431

28. Scott PH, Brunn GJ, Kohn AD, Roth RA, Lawrence JC Jr (1998) Evidence of insulin-stimulated phosphorylation and activation of the mammalian target of rapamycin mediated by a protein kinase B signaling pathway. Proc Natl Acad Sci USA 95:7772-7777

29. Hinault C, Mothe-Satney I, Gautier N, Lawrence JC Jr, Van Obberghen E (2004) Amino acids and leucine allow insulin activation of the $\mathrm{PKB} / \mathrm{mTOR}$ pathway in normal adipocytes treated with wortmannin and in adipocytes from $d b / d b$ mice. FASEB J 18:1894-1896

30. Pham P-TT, Heydrick SJ, Fox HL, Kimball SR, Jefferson LS Jr, Lynch CJ (2000) Assessment of cell-signaling pathways in the regulation of mammalian target of rapamycin (mTOR) by amino acids in rat adipocytes. J Cell Biochem 79:427-441
31. Giorgino F, Pedrini MT, Matera L, Smith RJ (1997) Specific increase in $\mathrm{p} 85 \alpha$ expression in response to dexamethasone is associated with inhibition of insulin-like growth factor-I stimulated phosphatidylinositol 3-kinase activity in cultured muscle cells. J Biol Chem 272:7455-7463

32. Ueki K, Yballe CM, Brachmann SM et al (2002) Increased insulin sensitivity in mice lacking $\mathrm{p} 85 \beta$ subunit of phosphoinositide 3-kinase. Proc Natl Acad Sci USA 99:419-424

33. Lamia KA, Peroni OD, Kim Y-B, Rameh LE, Kahn BB, Cantley LC (2004) Increased insulin sensitivity and reduced adiposity in phosphatidylinositol 5-phosphate 4kinase $\beta^{-}$mice. Mol Cell Biol 24:5080-5087

34. Luo J, Field SJ, Lee JY, Engelman JA, Cantley LC (2005) The p85 regulatory subunit of phosphoinositide 3-kinase downregulates IRS-1 signaling via the formation of a sequestration complex. J Cell Biol 170:455-464

35. Kirwan J, Varastehpour A, Jing M et al (2004) Reversal of insulin resistance post-partum is linked to enhanced skeletal muscle insulin signaling. $\mathrm{J}$ Clin Endocrinol Metab 89:4678-4684 